\title{
PREFACE
}

\section{A SPECIAL SELECTION ON BIOMECHANICAL APPLICATIONS IN MEDICAL SCIENCE -PART I}

Published 21 July 2021

This special selection on Biomechanical Applications in Medical Science presents the ongoing and active research in Biomechanics area. The overall goal is to summarize recent discoveries and groundbreaking studies that will account for new challenge research in the broad and relevant area of Biomechanics used for medical science. The details of the accepted 24 papers are highlighted as follows.

An et al. study the correlation between diaphragm excursion with both the quality of life and exercise capacity in patients with chronic obstructive pulmonary disease (COPD) by ultrasound, and to reveal the factors affecting diaphragm excursion. They conclude that the smaller the diaphragm excursion in patients with COPD the worse the quality of life and the lower the exercise capacity; and the decreased diaphragm excursion could serve as a factor for determining the exercise capacity impairment of COPD patients.

Park et al. compare the effects of gym ball exercise, with and without dynamic neuromuscular stabilization (DNS) core stability exercise, on spine kinematics and pain control in youth baseball player with scoliosis. The results provide novel, promising clinical evidence that DNS exercise rapidly improves Cobb's angles and relieve pain with improved cervical-thoracic-lumbar spinal stability.

Cai et al. assess the effectiveness of vibration in accelerating bone remodeling and orthodontic tooth movement. Databases of PubMed, Web of Science, and ScienceDirect are searched from January 2017 to March 2019 for randomized or quasi-randomized controlled trials that evaluate the effectiveness of vibration in accelerating bone remodeling and orthodontic tooth movement. Further research is necessary to determine the most feasible protocols for investigating the effects of magnitude and frequency of vibration on the acceleration of orthodontic tooth movement in human participants.

Cha et al. develop a smart auxiliary workwear and investigate its effectiveness through human impact assessment experiments. According to the biomechanical difference between squat and stoop motion, it is used as a stability maintenance strategy to increase the use of some muscles of the lower extremities in order to 
prevent fall due to body and weight. The proposed smart assistive work clothes could contribute to reduced muscle usage required for work and lessen related weariness by supporting the waist, and thus, would greatly help farmers in preventing musculoskeletal disorders.

Ko et al. investigate the test-retest reliability of static postural balance variables in natural and feet-together stance conditions. This study demonstrates that the mean velocity is the most reliable variable in both the natural and feet-together stance conditions. Also, the reliability of the natural stance is improved by repetition, whereas the reliability of the feet-together stance is not affected by the number of repetitions. The findings will contribute to the selection of reliable postural balance variables and protocols that researchers can use to assess effective balance performance in experimental studies of postural control.

Chen et al. discuss the discrepancy in output variables to the changes of element type and mesh density under barefoot and footwear conditions with compressive and shear loads. They find that universal optimal mesh criteria may not exist and convergence behavior of some output variables such as principal strains and vonMises stresses vary significantly among different mesh densities. The current study could offer a general guideline when generating a reasonable accurate finite element models for the analysis of plantar pressure distributions and stress/strain states employed for foot and footwear biomechanics evaluations.

Park et al. compare robot-interactive gait training (RIGT), with and without ankle control actuator, on ankle muscle activity and joint angle kinematics in healthy adults and participants with brain damage. They conclude that there are clinical evidence-based insights on the utilization of robot interactive locomotor training with ankle control to maximize and develop recovery of ankle-knee-hip joint movement control, thereby improving walking safety, preventing falls, and thus, improving the quality of life and the activities of daily living.

Jang et al. propose the detection of ventricular fibrillation (VF) using wavelet transform (WT) and phase space reconstruction (PSR) from electrocardiogram (ECG) signals. A neural network with weighted fuzzy memberships (NEWFM) is used to detect VF as a classifier. The performance results of the NEWFM exhibit a significant improvement in performance in terms of sensitivity, specificity, and accuracy. The technique proposed in this study implies that BSWFM can be set up in a portable automatic external defibrillator (AED) to detect VF in an emergency.

Zhong et al. present an integrated design of wearable sensors on a smart garment to concurrently measure the multi-channel electrocardiogram, respiration, and temperature signals in different regions of the body. This design shows excellent elasticity and consistent stretching performance to achieve close to clinical level signal quality under static and moderate body movement scenarios as being validated by measurements. An ultra-elastic structure of E-textile wire is proposed with excellent electrical stability, high stretch ratio, and low tension under body dynamics. The proposed design may constitute a general solution of distributed noninvasive physiological multi-parameter detection and monitoring applications. 
Choi et al. calculate the continuous relative phase (CRP) between joints. To do this calculus, the portrait method based on the joint angle and angular velocity and the Hilbert transform method based on the analytical signal have been widely used. They conclude with a high similarity and greater agreement between the portrait and Hilbert transform methods for the calculation of the CRP.

Yook et al. measure anthropometry, range of motion, and muscle strength of Koreans with paraplegia, using wheelchairs. For the research, people with paraplegia living in Chungcheongnam-do, Korea are recommended through the disability-related organizations. They conclude that the measurement results of Koreans with paraplegia would be used as basic data for designing and developing assistive technology products and universal products for wheelchair users and individuals who work and perform activities mainly in sitting posture.

Yoon et al. research the effect of patient-centered robot-assisted intervention for upper limb function improvement in stroke patients. The experimental group uses the COPM (Canadian Occupational Performance Measure) to identify subjectively preferred "patient-centered" focus need areas to perform robotic device aided daily activities. They conclude that the "patient-centered" approach significantly improves upper limb function and ADL (activities of daily living) performance compared to "robot-centered" rehabilitation. Further studies are warranted to confirm these results and for generalizability in clinical application.

Lin et al. work in the Taguchi-based optimization of head and neck CT angiography (CTA) using in vivo enhanced triggered timing for 600 patients. The signalto-noise ratio is defined as high LRA/US ratio (both left and right arterial pressure divided by upper sinuses' one) and low st-dev. They conclude that the successful factor combination of head and neck CTA scanning will also be recommended as a standard protocol for medical staff in deriving the maximal LRA/US value (both left and right arterial pressure divided by upper sinuses' one).

Choi et al. investigate neuromuscular mechanisms and effects by comparing the natural core stabilisation (NCS), abdominal bracing stabilisation (ABS), and coordinated core stabilisation (CCS) techniques in nonsymptomatic adults during lifting movement. They demonstrate superior biomechanical and physiological effects in CCS as compared with NCS and ABS during lifting movement in nonsymptomatic adults. Their novel results provide promising empirical evidence that CCS-based lifting movement is more balanced or coordinated in terms of neuromuscular control than other techniques and may be used as an alternative exercise for core stabilization.

Jeong et al. aim to investigate the effect of lower limb exercise on electroencephalogram (EEG) activity and posture stability during whole body vibration (WBV) for healthy elderly people. Subjects are composed of 20 elderly people who are over 60 years old and had no disease. They conclude that WBV stimulation significantly improves the postural stability, lower extremity muscles, and brain activation of the elderly. Moreover, it significantly reduces the total time required for the timed up and go (TUG) test and facilitates the activation of lower extremity 
muscle. These results may be helpful to protocol of exercise using WBV for the healthy elderly.

Park et al. compare the effects between tablet computer-based productive and receptive cognitive engagement using an alternating-treatment design. The productive engagement is associated with higher prefrontal cortex activity and faster performance in the Trail Making Test, compared to those utilizing the receptive engagement. These results suggest that the productive engagement might be effective in facilitating prefrontal cortex activity and improving executive function of healthy older adults, indicating cognitively challenging activities are more beneficial relative to nonchallenging activities.

Hong et al. identify factors associated with the digital healthcare needs of people with severe disabilities in ADL (activities of daily living). Qualitative data are collected from in-depth individual interviews and focus group interviews from 16 participants. Authors conclude that digital healthcare could help support the continued and appropriate use of healthcare for people with severe disabilities over the long term. In addition, they can create and provide digital healthcare technology that people with severe disabilities need and can operate.

$\mathrm{Li}$ et al. analyze the correlation between the vascular endothelial function (characterized by endothelin-1 (ET-1) and nitric oxide (NO)) and the renal hemodynamics in patients with hypertensive disorders in pregnancy (HDP) by color Doppler ultrasound. Indicators of renal hemodynamics measured by color Doppler ultrasound are correlated to the serum levels of ET-1 and NO characterizing the vascular endothelial function. They are sensitive indicators reflecting hemodynamic changes and renal impairment in HDP patients.

Luo et al. propose that the total energy the brain consumes and the intensities of information flows across different brain regions in an intellectual activity may help to explain an individual's intelligence level. To verify this assumption, 43 students aged 18-25 are recruited as the research subjects. They conclude that PSI (Phase Slope Index) values in different channel combinations are used to demonstrate the efficiency of the brain network in accordance with the IQ (intelligence quotient) scores. It is found that the energy of EEG (electroencephalogram) signals in an intellectual activity is negatively correlated with the IQ score.

Kim et al. evaluate the difference of proprioception in standing and sitting postures through joint position error test. Authors conclude that, in sitting posture, posterior neck muscles are used more than in standing posture, which may negatively affect the proprioceptive accuracy of the neck and may also increase the neck repositioning errors. The results of this study may be helpful as a basic data for treating musculoskeletal disorders associated with abnormal neck position in the clinic, such as the forward neck position.

Park et al. compare the effects of the dynamic neuromuscular stabilization (DNS) breathing technique and the abdominal bracing technique $(\mathrm{AB})$ on upper trapezius (UT), anterior deltoid (AD), pectoralis major (PM), bilateral $\mathrm{IO} / \operatorname{Tr} \mathrm{A}$ and bilateral external oblique (EO) motor control in healthy participants during shoulder 
horizontal adduction with loads. Paired $t$-tests are used to analyze electromyography (EMG) data to determine statistically significant differences in muscle activity between the two techniques. The results provide clinical evidence that core exercise with DNS technique is more effective in lessening UT, AD, and PM muscle activation as well as improving the bilateral TrA/IO motor control in participants.

Kwon et al. put forward quantitatively clinical characteristics of action tremor during spiral drawing task using 3-axis gyro sensors. 18 essential tremor patients with sensors attached on their forefinger, dorsum of hand and forearm participated in a spiral drawing test. The logarithmic variables from gyro sensors show main and interactive effects of joint (sensor attachment location) and direction (pitch, yaw, and roll). Significant differences are observed among joints particularly in roll direction. Kinetic tremor is prominent mainly in the roll direction and in the finger joint.

Oh et al. confirm an effective recovery method on muscle function and blood of lactate level after maximal isotonic exercise. Sling exercise therapy using supine position and whole-body vibration of $10 \mathrm{~Hz}, 5 \mathrm{~mm}$ are performed. Lactate level and muscle function during the rest, fatigue and recovery period are measured. And then maximal exercise using back extension is performed to induce fatigue. This study demonstrates that sling exercise therapy with intermittent whole-body vibration could provide positive effect on efficient recovery of muscle function and higher reduction of lactate after maximal isotonic exercise.

Liu et al. use maternal-fetal Holter monitor which is based on the technology of fetal electrocardiograph (FECG) to collect fetal heart rate (FHR), and then design algorithm to extract the baseline FHR, acceleration, variation, sleep-wake cycle, and nonlinear parameters. More parameters of FHR are explored to be used to evaluate the state of fetus. They conclude with the proposition of a classification method for prenatal fetal monitoring with high accuracy.

To sum-up, this current special issue is a continuous effort in promoting the biomechanical application in medical diagnosis and treatment, $, 1,2,8,12,13,17,18,21,22$ including rehabilitation engineering, finite element modeling, and biofluid mechanics, etc. in conjunction with Biomedical Imaging. ${ }^{3-7,9-11,14-16,19,20}$ As guest editors, we sincerely hope that the selected papers will be of long-lasting value to biomechanics, and we believe that it could allow some insights for the challenging field of Biomechanical Applications in Medical Science and relevant areas.

$$
\begin{array}{r}
\text { Guest Editors } \\
\text { Esteban Peña Pitarch } \\
\text { Universitat Politècnica de Catalunya }(U P C) \text {, Spain }
\end{array}
$$

Eddie Y. K. Ng Nanyang Technological University, Singapore 


\section{References}

1. Liu F, Ng EYK, Zi Chen, A special section on biological mechanics, J Mech Med Biol 15(6):1502002-1-1502002-3, 2015.

2. Liu F, Ng EYK, A special section on biological mechanics, J Mech Med Biol 16(8):1602002-1-1602002-4, 2016.

3. Liu F, Ng EYK, A special section on biomedical imaging in diagnosis and treatment (Part 1), J Med Imag Health Inform 6(5):1209-1211, 2016.

4. Liu F, Ng EYK, A special section on biomedical imaging in diagnosis and treatment (Part 2), J Med Imag Health Inform 16(7):1670-1672, 2016.

5. Liu F, Ng EYK, A special section on biomedical imaging in diagnosis and treatment (Part 3), J Med Imag Health Inform 17(1):126-128, 2017.

6. Liu F, Ng EYK, A special section on methods and application in biomedical imaging (Part 1), J Med Imag Health Inform 7(5):919-921, 2017.

7. Liu F, Ng EYK, A special section on methods and application in biomedical imaging (Part 2), J Med Imag Health Inform 7(7):1522-1524, 2017.

8. Liu F, Ng EYK, A special section on biological mechanics, J Mech Med Biol 17(7):1702002-1-1702002-7, 2017.

9. Liu F, Ng EYK, A special section on methods and application in biomedical imaging (Part 3), J Med Imag Health Inform 8(1):1-4, 2018.

10. Gomez L, Ng EYK, A special section on methods and application in biomedical imaging (Part 1), J Med Imag Health Inform 8(7):1364-1367, 2018.

11. Gomez L, Ng EYK, A special section on methods and application in biomedical imaging (Part 2), J Med Imag Health Inform 8(8):1607-1610, 2018.

12. Peña E, Drochon A, Ng EYK, A special selection on biological applications of mechanics, J Mech Med Biol 18(7):1802001-1-1802001-8, 2018.

13. Peña E, Drochon A, Ng EYK, A special selection on biological applications of mechanics, J Mech Med Biol 18(8):1802002-1-1802002-8, 2018.

14. Gomez L, Ng EYK, A special section on methods and application in biomedical imaging (Part 3), J Med Imag Health Inform 9(1):43-46, 2019.

15. Gomez L, Ng EYK, A special section on methods and application in biomedical imaging (Part 1), J Med Imag Health Inform 9(7):1415-1417, 2019.

16. Gomez L, Ng EYK, A special section on methods and application in biomedical imaging (Part 2), J Med Imag Health Inform 9(9):1849-1852, 2019.

17. Peña E, Drochon A, Ng EYK, A special selection on biomechanics in medical application (Part 1), J Mech Med Biol 19(7):1902003-1-1902003-8, 2019.

18. Peña E, Drochon A, Ng EYK, A special selection on biomechanics in medical applications (Part 2), J Mech Med Biol 19(8):1902004-1-1902004-8, 2019.

19. Gomez L, Ng EYK, A special section on emerging techniques for biomedical imaging (Part 3), J Med Imag Health Inform 10(3):610-613, 2020.

20. Gomez L, Ng EYK, A special section on emerging techniques for biomedical imaging (Part 1), J Med Imag Health Inform 10(11):2660-2662, 2020.

21. Peña E, Drochon A, Ng EYK, A special selection on mechanical engineering applied to biomedicine (Part 1), J Mech Med Biol 20(9):2002001-1-2002001-7, 2020.

22. Peña E, Drochon A, Ng EYK, A special selection on mechanical engineering applied to biomedicine (Part 2), J Mech Med Biol 20(10):2002002-1-2002002-8, 2020. 